\title{
The Polycomb Protein Ezh2 Impacts on Induced Pluripotent Stem Cell Generation
}

\author{
Xiaolei Ding, ${ }^{1,2}$ Xiaoying Wang, ${ }^{1,2}$ Stephanie Sontag, ${ }^{1,2}$ Jie Qin, ${ }^{1,2}$ Paul Wanek,, \\ Qiong Lin, ${ }^{1,2}$ and Martin Zenke ${ }^{1,2}$
}

Reprogramming of somatic cells toward pluripotency involves extensive chromatin reorganization and changes in gene expression. Polycomb group (PcG) proteins are key regulators of chromatin structure, cell identity, and development. In this study, we investigated the impact of Ezh2, a core subunit of Polycomb repressive complex 2 (PRC2), on the generation of induced pluripotent stem (iPS) cells. We found that Ezh2 expression is induced during iPS cell generation and iPS cells contain high levels of Ezh2 mRNA and protein. Importantly, shRNA knockdown of Ezh2 during reprogramming severely impairs iPS cell generation. Mechanistically, Ezh2 acts during reprogramming at least in part through repressing the Ink4a/Arf locus, which represents a major roadblock for iPS cell generation. Interestingly, knockdown of Ezh2 in established pluripotent cells leaves pluripotency and self-renewal of embryonic stem cells and iPS cells unaffected. Altogether, our results demonstrate that Ezh2 is critical for efficient iPS cell generation, whereas it is dispensable for maintaining the reprogrammed iPS cell state.

\section{Introduction}

$\mathrm{P}$ OLYCOMB GROUP (PcG) PROTEINS form two large multiprotein complexes, referred to as Polycomb repressive complex 1 and 2 (PRC1 and PRC2, respectively), which impact on histone modification, chromatin structure, and gene expression during development [1-4]. PcG proteins are conserved from Drosophila to human and involved in maintaining cellular memory and silencing gene expression. PRC2 contains Ezh2, Eed, and Suz12 proteins, and trimethylates histone 3 lysine 27 (H3K27me3), which is implicated in silencing gene expression. PRC1 contains Bmi1, Ring1A/B, Cbx, Mel18, and Mph and is recruited to specific sites formed by PRC2, referred to as maintaining complex [1-4]. PRC1 and PRC2 are involved in various biological processes, including stem cell self-renewal, commitment and differentiation, and in maintaining cell identity and also in cancer cell formation [2]. In embryonic stem (ES) cells, a subset of chromatin regions of development-associated genes is characterized by $\mathrm{H} 3 \mathrm{~K} 27 \mathrm{me} 3$, which is catalyzed by PRC2 and correlates with gene silencing $[5,6]$. In loss-of-function studies, for example in Ezh2, Eed, and Suz12 null ES cells, such silent genes are derepressed. Furthermore, PcG protein deficiencies lead to severe defects in ES cell differentiation, emphasizing their essential role in maintaining an ES cellspecific gene expression repertoire and in executing development programs during ES cell differentiation $[5,6]$.
PcG proteins are also required for establishing ES cell lines and for reprogramming somatic cells toward pluripotency. For example, blastocysts deficient for the PRC2 component Ezh2 failed to yield ES cells or produced ES cells at very low frequency $[7,8]$. ES cells lacking the PRC2 components, Ezh2, Eed, and Suz12, were deficient in cell fusion-induced reprogramming of somatic cells toward pluripotency [9]. In somatic cell nuclear transfer (SCNT) experiments, the inner cell mass of cloned embryos showed low H3K27me3 modification compared to fertilized embryos and thus differentiation-related genes were expressed [10]. Furthermore, the low levels of H3K27me3 in SCNT embryos correlate with low Ezh2 expression in such cloned embryos. All these studies support the notion that PcG proteins contribute to establish pluripotency.

Induced pluripotent stem (iPS) cells are generated from somatic cells by transduction of specific reprogramming transcription factors [11]. iPS cells hold great potential in disease modeling, drug discovery, and cell-based therapies $[12,13]$. iPS cell generation is regulated by a series of complex processes that are increasingly being better understood [14-19]. Extensive epigenetic reorganization occurs during reprogramming, and recent studies indicate that activities of epigenetic modifiers play an important function in reprogramming, and thus, the role of $\mathrm{PcG}$ proteins in iPS cell generation is now beginning to be studied in detail [15,19-24].

\footnotetext{
${ }^{1}$ Department of Cell Biology, Institute for Biomedical Engineering, RWTH Aachen University Medical School, Aachen, Germany.

${ }^{2}$ Helmholtz Institute for Biomedical Engineering, RWTH Aachen University, Aachen, Germany.
} 
Here, we investigated the impact of the PcG protein Ezh2 on iPS cell generation. We studied the influence of Ezh2 overexpression and knockdown on iPS cell generation. We show that Ezh2 is critical for efficient iPS cell generation and acts-at least in part-through repressing the cell cycle regulator Ink4a/Arf.

\section{Materials and Methods}

\section{Cells and cell culture}

Mouse embryonic fibroblasts (MEF) were isolated from C57BL/6 mice or Oct4-eGFP transgenic OG2 mice [25]. MEF and 293T cells were cultured in Dulbecco's modified Eagle's medium (DMEM; Invitrogen), containing 10\% FCS, $2 \mathrm{mM}$ L-glutamine, and 100 units penicillin $/ 100 \mu \mathrm{g}$ streptomycin.

ES cells (HM1) and iPS cells were cultured, as described previously [26,27]. Briefly, cells were maintained on inactivated MEF feeder layer from C57BL/6 mice with ES cell medium. The ES cell medium was DMEM (high glucose), supplemented with $15 \%$ heat-inactivated fetal calf serum (FCS; Lonza), $25 \mathrm{mM}$ HEPES, and 1,000 U recombinant leukemia inhibitory factor, $2 \mathrm{mM}$ L-glutamine, 100 units penicillin $/ 100 \mu \mathrm{g}$ streptomycin, $0.1 \mathrm{mM}$ nonessential amino acids, and $50 \mu \mathrm{M} \beta$-mercaptoethanol. Cells were cultured at $37^{\circ} \mathrm{C}$ in a $5 \% \mathrm{CO}_{2}$ incubator. The medium was refreshed daily, and cells were passaged every 2-3 days before reaching confluency. Unless stated differently, all reagents were from Invitrogen.

\section{Plasmids, virus preparation, and iPS cell generation}

pMX retrovirus vectors with reprogramming factors, Oct4, Sox2, Klf4, and c-Myc, were used for iPS cell generation [11,28]. MSCV retrovirus vector containing mouse Ezh2 cDNA was obtained from Addgene (www .Addgene.org), as described previously [29]. Retrovirus vector with short hairpin RNA (shRNA) for Ink4a/Arf was kindly provided by $\mathrm{H}$. Li and M. Serrano, as described previously [30,31]. Lentivirus eGFP vectors with shRNA targeting Ezh2 (shEzh2-1, shEzh2-2) and shLuc targeting luciferase were kindly provided by A. Iwama [32].

For virus preparation, 293T cells were transfected with vector constructs and packaging plasmids (pVPack-GP and pVPack-Eco; Stratagene). shRNA lentivirus vectors targeting Ezh2 were transfected into $293 \mathrm{~T}$ cells with the packaging plasmids pCMV $\Delta$ R8.74 and pVSV-G, as described previously [26]. Transfections were with the calcium phosphate coprecipitation method. Forty-eight hours after transfection, virus supernatant was harvested and concentrated by precipitation with chondroitin 6-sulfate (CSC) and polybrene (both Sigma) and centrifuged [33].

For iPS cell generation, $5 \times 10^{4} \mathrm{MEF}$ of C57BL/6 mice or Oct4-eGFP transgenic OG2 mice were seeded per well of six-well plate the day before infection and exposed to concentrated pMX viruses with Oct4, Sox2, Klf4, and $\mathrm{c}-\mathrm{Myc}$ for $24 \mathrm{~h}$ and were cultured in the ES cell medium. For OG2 $\mathrm{MEF}$ as targets, GFP-positive $\left(\mathrm{GFP}^{+}\right)$colonies appeared around day 14 after virus transduction. $\mathrm{GFP}^{+}$iPS cells were picked at day 18 and transferred into 96-well format. For C57BL/6 MEF, kinetics of iPS cell generation was similar to OG2 MEF iPS cells, and emerging iPS cells were identified by morphology and picked 2-3 weeks after infection. Single cell suspensions were obtained by trypsin treatment, and cells were cultured in 24-well plates and further in 6-well plates on MEF feeder in the ES cell medium.

\section{Alkaline phosphatase staining}

Emerging iPS cells were subjected to alkaline phosphatase (AP) staining by using the Alkaline Phosphatase Staining Kit (Stemgent) according to the manufacturer's protocol. Stained cells were photographed, and $\mathrm{AP}^{+}$colonies were scored.

\section{Cell cycle analysis and $\beta$-galactosidase staining}

For cell cycle analysis, cells were fixed, incubated with 7 -aminoactinomycin D (7-AAD) $(10 \mu \mathrm{g} / \mathrm{mL}$; Molecular Probes, Invitrogen), as described previously [34] and submitted to the flow cytometry analysis (FACSCanto device; BD Biosciences). Data were analyzed with FlowJo software (Tree Star).

MEF were measured for the $\beta$-galactosidase ( $\beta$-gal) activity using the $\beta$-gal staining kit (Cell Signaling Technology).

\section{$D N A$ and RNA isolation and polymerase chain reaction}

For DNA isolation, the NucleoSpin Tissue Kit (MachereyNagel) was used and $50 \mathrm{ng}$ total DNA was subjected to quantitative polymerase chain reaction (qPCR) with SYBR Green PCR master mix and eGFP-specific primers (see Supplementary Table S1;Supplementary Data are available online at www.liebertpub.com/scd) employing the Applied Biosystems 7300 Real-Time PCR system (Applied Biosystems).

Total RNA was isolated with the RNeasy Mini Kit (Qiagen). One microgram of total RNA was used for cDNA preparation with the High Capacity cDNA Reverse Transcription Kit (Applied Biosystems). cDNA was then used for PCR amplification by Taq DNA polymerase (Fermentas Life Sciences). PCR fragments were separated on $2 \%$ agarose gels, and images were recorded with the Gel-Doc system (BioRad).

Quantitative real time-PCR (qRT-PCR) was carried out with the Applied Biosystems 7300 Real-Time PCR system (Applied Biosystems). Reactions were performed with $50 \mathrm{ng}$ cDNA, SYBR Green PCR master mix, and primers (see Supplementary Table S1). The calculated threshold cycle (CT) value for each sample was normalized against the corresponding $\beta$-actin $C_{T}$ value.

\section{Western blotting and immunofluorescence}

Cells were lysed in $2 \%$ SDS with $5 \mathrm{mM}$ EDTA. Twenty micrograms of protein lysate was subjected to SDS-PAGE (12\% SDS-polyacrylamide gels) and western blotting. Membranes were blocked overnight with $2 \%$ nonfat milk in PBS at $4^{\circ} \mathrm{C}$ and incubated with primary antibodies (mouse anti-Ezh2, AC22, 1:1,000, Cell Signaling Technology; rabbit polyclonal anti-H3K27me3, 1:100, Millipore; mouse anti-Oct3/4, C-10, 1:200, Santa Cruz Biotechnology; mouse anti-actin antibody, clone AC-74, 1:5,000, Sigma) at room temperature for $2 \mathrm{~h}$. Peroxidase-conjugated secondary antibodies were incubated for $1 \mathrm{~h}$ at room temperature and subjected to chemiluminescence (SuperSignal $^{\circledR}$; Thermo Scientific). 
For immunofluorescence staining, ES cells and iPS cells were grown on gelatin-coated chamber slides, fixed with $1 \%$ formaldehyde, and permeabilized with PBS buffer containing $0.5 \%$ BSA and $0.1 \%$ Triton X-100. Cells on coverslips were washed two times with $0.5 \%$ BSA in PBS buffer and then incubated with primary antibodies (mouse anti-Ezh2, clone AC22, 1:100, Cell Signaling Technology; mouse antiSSEA-1, clone 480, 1:50, Santa Cruz Biotechnology; and mouse anti-Oct3/4, clone C-10, 1:100, Santa Cruz Biotechnology). Secondary antibody was anti-mouse Alexafluor647 (Invitrogen), and DAPI was used to stain nuclei. Coverslips were mounted onto glass slide with the mounting medium, and images were acquired under bright and fluorescent fields with an Axiovert 200 microscope (Carl Zeiss).

\section{Teratoma formation assay}

NOD/SCID mice were maintained under specific pathogen-free conditions in the central animal facility of the RWTH Aachen University Hospital. All animal experiments were approved by local authorities in compliance with the German animal protection law.

For teratoma formation assay, $2 \times 10^{6}$ iPS cells were injected into the rear thigh of NOD/SCID mice. HM1 ES cells were used as control. After 1 month, mice were sacrificed, teratomas excised, fixed in $4 \%$ paraformaldehyde, embed- ded in paraffin, sectioned, and analyzed after hematoxylin and eosin (HE) staining.

\section{Statistics}

Data are given as the means \pm standard error of the mean (SEM). Student's $t$-test was used for comparison of pairs of differences, and statistics of $P<0.05$ were considered significant.

\section{Results}

\section{Ezh2 expression increases during iPS cell generation}

ES cells and iPS cells express various PcG components (Fig. 1A, B and Supplementary Fig. S1A, B). For example, ES cells and iPS cells derived from MEF, neural stem cells (NSC), and hematopoietic stem cells (HSC) express high levels of Ezh2. MEF and HSC, which were used for reprogramming, express low Ezh2 levels. NSC contain rather high levels of Ezh2, which might be related to their high proliferation potential. Additionally, NSC are also readily reprogrammed into iPS cells by two factors (Oct4 and Klf4) only [28]. iPS cell lines differed in Ezh2 expression, which was related to the cell type used for reprogramming and/or due to clonal variation. Western blot analysis extended the Ezh2 RNA expression data and
A
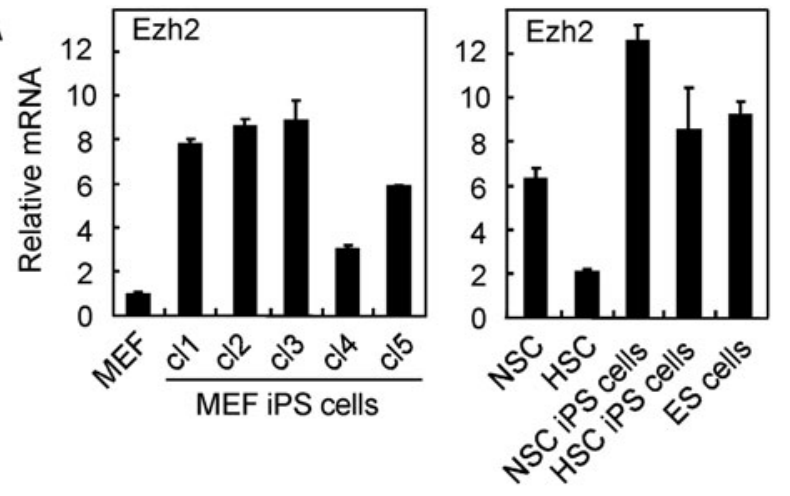

B

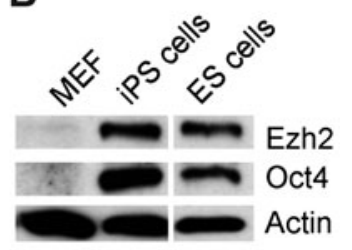

C

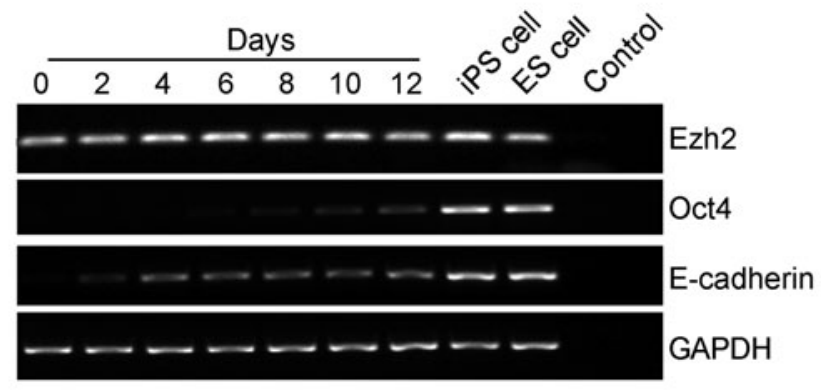

D
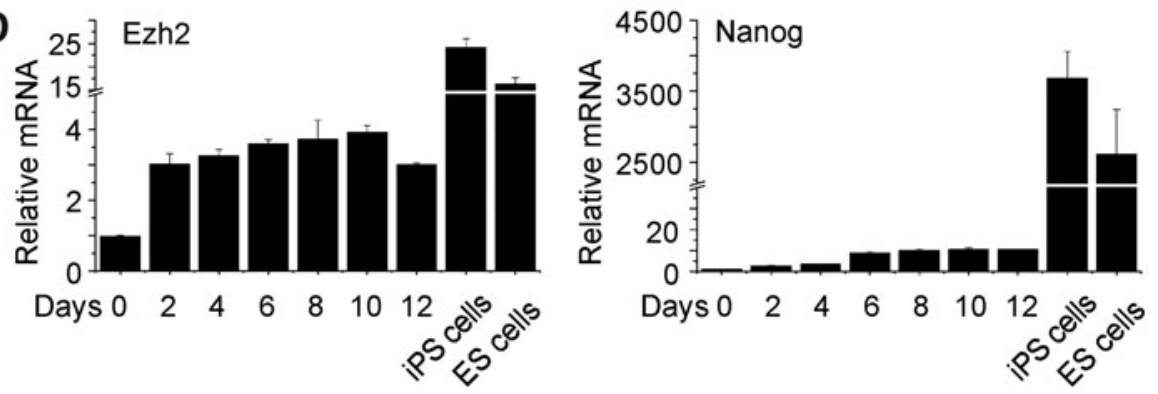

FIG. 1. Ezh2 expression is induced during induced pluripotent stem (iPS) cell generation. (A) Ezh2 RNA expression in mouse embryonic fibroblasts (MEF), neural stem cells (NSC), hematopoietic stem cells (HSC), iPS cells, and embryonic stem (ES) cells was measured by qRT-PCR. Expression levels were normalized to $\beta$-actin. Ezh2 expression in MEF was set as a reference to 1.0 , and relative RNA levels are shown. MEF iPS cells (clones cl1-5), NSC iPS cells, and HSC iPS cells refer to iPS cells derived from MEF, NSC, and HSC, respectively. (B) Ezh2 protein expression in MEF, MEF iPS cells, and ES cells was determined by western blotting with Ezh2-specific antibody. The pluripotency marker Oct 4 and loading control $\beta$-actin are shown. (C) RT-PCR analysis of Ezh2, Oct4, and E-cadherin expression during iPS cell generation (day 0-12) and in established iPS cells and ES cells. GAPDH was used as loading control. MEFs were infected with the reprogramming factors, Oct4, Sox2, Klf4, and cMyc, and RNA was obtained after various periods of time as indicated and analyzed. Control, no cDNA input. (D) Kinetics of Ezh2 and Nanog RNA expression during iPS cell generation as in (C) (day 0-12) by qRT-PCR and normalized to $\beta$-actin. 
showed very low Ezh2 expression in MEF and high Ezh2 expression in ES cells and iPS cells (Fig. 1B).

iPS cell generation entails the repression of somatic genes and activation of pluripotency genes, such as Oct4 and Nanog. To measure Ezh2 expression during reprogramming, MEF were transfected with the reprogramming factors, Oct4, Sox2, Klf4, and c-Myc (OSKM), and RNA was isolated in regular time intervals. The kinetics of Ezh2 expression was analyzed by RT-PCR and related to the expression of Oct4, Nanog, and E-cadherin (Fig. 1C, D). Ezh2 mRNA is already expressed in MEF and further increased during reprogramming, and thus, established iPS cell lines showed high Ezh2 expression. E-cadherin, an early marker for reprogramming, was already observed at day 2, whereas the pluirpotency markers Oct4 and Nanog were detected from day 6-8 onward.

These data indicate that during iPS cell generation Ezh2 expression is induced and its expression is high in established iPS cells. The increase of Ezh2 expression during reprogramming led us to investigate whether Ezh2 plays an active role in iPS cell generation.

\section{Forced Ezh2 expression enhances reprogramming efficiency}

To investigate the impact of forced Ezh2 expression on iPS cell generation, Oct4-eGFP MEF from OG2 mice [25] were infected with OSKM reprogramming factors plus Ezh2 using retroviral vectors. Infected cells were maintained in the ES cell culture medium, and Oct4-GFP ${ }^{+}$colonies appeared around day 15 after infection, both with and without Ezh2 (Fig. 2A). Interestingly, forced Ezh2 expression caused about 1.5- to 2-fold higher frequency of Oct4-GFP ${ }^{+}$cell and iPS cell colonies (Fig. 2A-D). The increase in iPS cell colony formation did, however, not reach statistical significance, probably due to variation in colony formation, which is frequently observed for primary iPS cells. Ezh2-iPS cells displayed typical ES cell morphology and growth characteristics and expressed the pluripotency markers, Oct4, Sox2, Nanog, Dppa4, and SSEA1 (Fig. 2E, F). Finally, Ezh2-iPS cells were injected into NOD-SCID mice and analyzed for teratoma formation. Ezh2-iPS cells developed teratomas in recipient mice, which were of comparable size as those of ES cells and iPS cells without Ezh2 (data not shown). Histologically, these teratomas contained cell derivatives of all three germ layers (Supplementary Fig. S2).

In summary, forced Ezh2 expression enhanced the fourfactor induced iPS cell generation to some extent, and such Ezh2-iPS cells showed biological properties similar to iPS cells without ectopic Ezh2 expression.

\section{c-Myc induces Ezh2 expression, but Ezh2 cannot compensate for c-Myc in iPS cell generation}

MEF lose their proliferative capacity with extended periods of time in culture and acquire a senescent phenotype,
FIG. 2. Forced Ezh2 expression enhances the efficiency of iPS cell generation. Generation of iPS cells from Oct4-eGPF OG2 MEF with forced Ezh2 expression. OG2 MEF were transduced with the four reprogramming factors (Oct4, Sox2, Klf4, and c-Myc, OSKM) plus Ezh2, or empty vector control (vector). (A) Images show representative $\mathrm{GFP}^{+}$iPS cell colonies at day 16 after infection. Scale bars, $100 \mu \mathrm{m}$. (B) Quantification of $\mathrm{GFP}^{+}$iPS cell colony numbers at day 20. Data represents mean values of six independent experiments $(P=0.1898)$. (C) FACS analysis of $\mathrm{GFP}^{+}$cells as in (A) at day 25 (Ezh2) and empty vector control (vector). (D) Percentage of $\mathrm{GFP}^{+}$ cells (mean values of three independent experiments). (E) Ezh2-iPS clones: morphology and GFP expression of Ezh2-iPS cell clones (upper panel). Immunofluorescence analysis of the pluripotency markers, Oct4 and SSEA1 (lower panel). Scale bars, $100 \mu \mathrm{m}$. (F) RT-PCR analysis of pluripotency genes (Oct4, Sox2, Nanog, Dppa4, and Rex1) in Ezh2-iPS clones E3, E7, $\mathrm{E} 8$, and E10. ES cells are shown as control and GAPDH serves as a loading control.
A
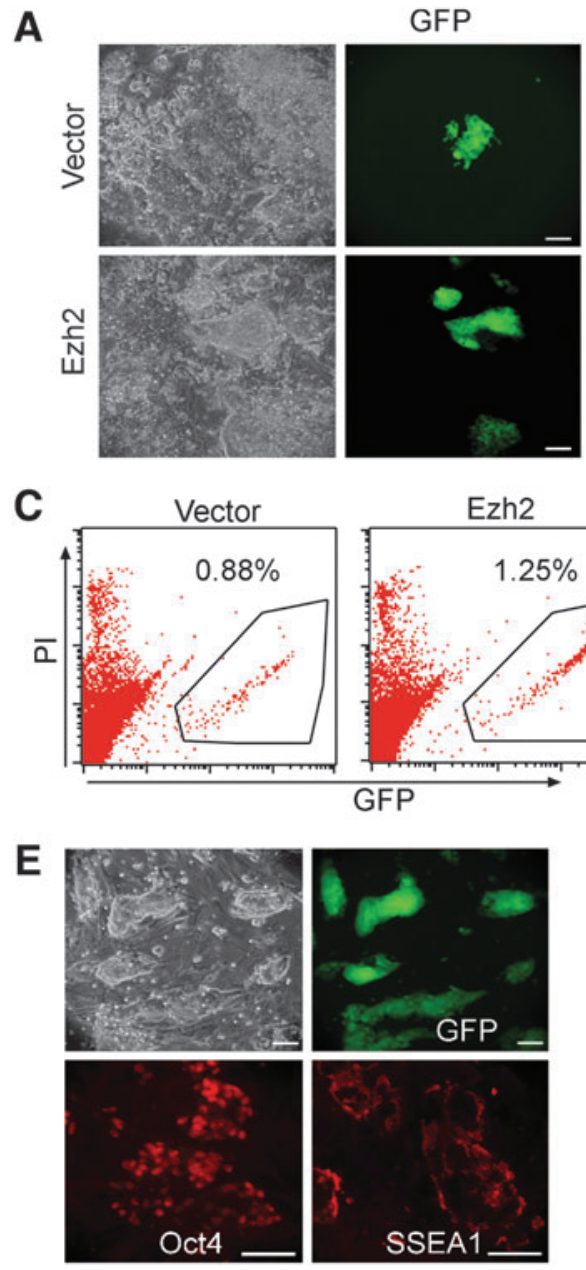

B

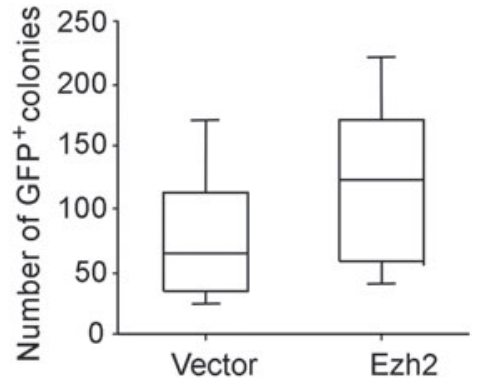

D

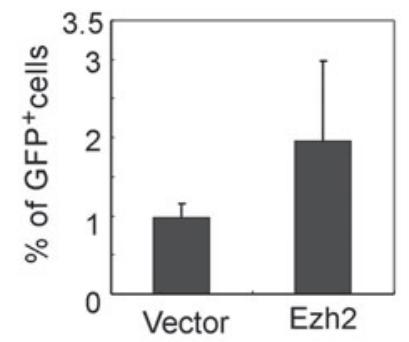

F Ezh2 iPS cells $\begin{array}{llll}\text { ES cells E3 } & \text { E7 } & \text { E8 } & \text { E10 }\end{array}$

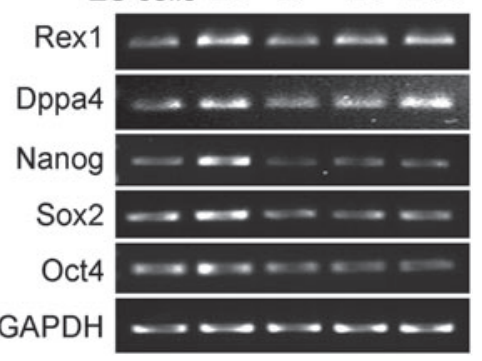


which is accompanied with the loss of Ezh2 expression [35]. shRNA-mediated Ezh2 downregulation also induces senescence (Supplementary Fig. S3D). Conversely, ectopic expression of Ezh2 overcomes senescence in MEF [35]. iPS cells show high Ezh2 expression (Fig. 1A-D) and also overcome senescence [36]. Therefore, iPS cell generation appears to be linked to high Ezh2 expression. Thus, to determine which reprogramming factor induces Ezh2 during iPS cell generation, MEF cells were transduced with individual reprogramming factors (Oct4, Sox2, Klf4, or c-Myc), and Ezh2 mRNA was determined at day 6 by qRT-PCR. Ezh2 expression was significantly upregulated in c-Myc-transduced cells, whereas cells transduced with the four reprogramming factors showed highest Ezh2 expression (Fig. 3A).

c-Myc is important for efficient generation of iPS cells, and thus, the question was whether forced expression of Ezh2 can substitute for c-Myc in iPS cell generation. To this end, iPS cells were generated from MEF with the three reprogramming factors, Oct4, Sox, and Klf4 (3F), with and without Ezh2 (Fig. 3B, C). Frequencies of alkaline phosphatase-positive $\left(\mathrm{AP}^{+}\right)$cells at day 10 after transduction were reduced without c-Myc, yet forced Ezh2 expression could not substitute for c-Myc (Fig. 3B, C). Essentially, the same result was obtained when Oct4-eGFP MEF of OG2 mice were used for reprogramming with Oct4, Sox2, and Klf4 (3F) with and without Ezh2. The frequencies of Oct4-
$\mathrm{GFP}^{+}$cells at day 25 after transduction were only slightly increased with forced Ezh2 (Fig. 3D).

Thus, c-Myc induces Ezh2 expression, yet forced Ezh2 expression cannot substitute for c-Myc in efficient iPS cell generation.

\section{Ezh2 is required for efficient iPS cell generation}

Ezh2 is important for establishing pluripotency. For example, (i) Ezh2-deficient mice do not allow the generation of ES cell lines or allow their generation only at very low efficiency and (ii) Ezh2-deficient ES cell lines failed in reprogramming B cells toward pluripotency by cell fusion [7,9]. In addition, as reported here, Ezh2 expression is robustly upregulated during iPS cell generation (Fig. 1A-D). Thus, we used shRNA-mediated knockdown of Ezh2 to determine whether Ezh2 is required for iPS cell generation. Lentivirusmediated shRNA targeting of Ezh2 caused effective downregulation of Ezh2 expression in MEF, as determined by qRT-PCR (Supplementary Fig. S3A). Concomitantly with Ezh2 knockdown, MEF ceased proliferating, showed a lower frequency of cells in $\mathrm{S}+\mathrm{G} 2$ phase, and acquired a senescent phenotype (Supplementary Fig. S3B-D).

To determine the impact of Ezh2 on iPS cell generation, $\mathrm{MEF}$ were infected with retroviruses containing the four reprogramming factors OSKM together with either Ezh2
A

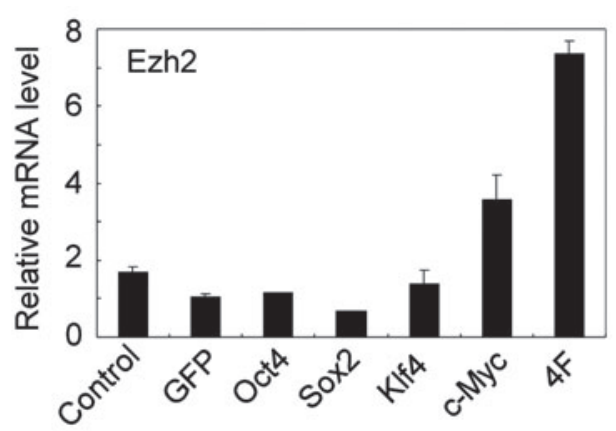

C

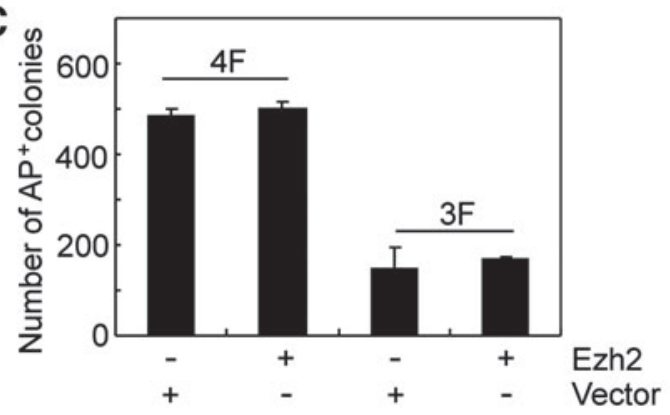

B
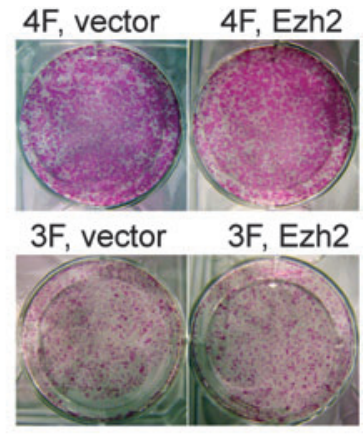

D

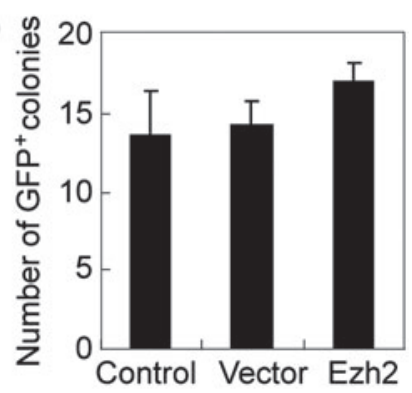

FIG. 3. c-Myc induces Ezh2 expression, but Ezh2 cannot replace c-Myc during iPS cell generation. (A) MEF were transduced with retroviral vectors containing the individual reprogramming factors, Oct4, Sox2, Klf4, or c-Myc, or transduced with all four factors (4F). eGFP vector was used as control (GFP). Untreated cells served as a further control (control). Ezh2 expression at day 6 after transduction was measured by quantitative real time (qRT-PCR) and normalized to $\beta$-actin. Ezh2 expression in GFP control was set as 1. (B) MEF were transduced with four reprogramming factors (Oct4, Sox2, Klf4, and c-Myc, 4F) and Ezh2 or with three factors (Oct4, Sox2, and Klf4, 3F) and Ezh2. Empty vector was used as control (vector). Representative images of alkaline phosphatase-positive $\left(\mathrm{AP}^{+}\right)$colonies at day 10 after infection are shown. (C) Numbers of $\mathrm{AP}^{+}$colonies of (B) of two independent experiments are shown. (D) Number of GFP ${ }^{+}$colonies. Oct4eGFP OG2 MEF were transduced with three reprogramming factors (Oct4, Sox2, and Klf4) with Ezh2 or empty vector control. Transduced cells were maintained in the ES cell medium, and the numbers of $\mathrm{GFP}^{+}$colonies were scored at day 25 . Data represent mean values of three independent experiments. 
shRNA hairpins (shEzh2-1, shEzh2-2) or control shRNA (shLuc; Fig. 4A, B). Ezh2 expression was efficiently silenced by Ezh2 shRNA, which led to a reduction in global H3K27me3 levels as demonstrated by western blotting (data not shown). At day 10, cells were stained for AP, which represents an early marker for iPS cell generation. Cells infected with shLuc control generated frequencies of $\mathrm{AP}^{+}$ colonies similar to control. Importantly, Ezh2 downregulation caused a strong reduction of $\mathrm{AP}^{+}$colonies (Fig. 4A, B). At day 20, we scored cultures for iPS cell colonies by morphology and observed a prominent reduction in iPS cell colony numbers by shEzh2-1 and shEzh2-2 compared to controls (Fig. 4C).

We next wanted to clarify why some iPS cells developed and whether these were refractory to shRNA-mediated Ezh2 knockdown. Therefore, iPS cells were generated with OSKM retroviruses together with either shEzh2 GFP vector (shEzh2-1, shEzh2-2) or control shLuc GFP vector, as described above. A panel of iPS cell clones refractory to shEzh2 was isolated and expanded. All iPS cell clones generated in the presence of shEzh2 GFP vector were GFP negative by fluorescence microscopy, indicating that they did not take up or express shEzh2 GFP vector and thus escaped Ezh2 knockdown. iPS cell clones with shLuc GFP vector were $\mathrm{GFP}^{+}$. Next DNA and RNA were isolated and analyzed for shEzh2 GFP vector sequences and vector expression by PCR. We found that all iPS cell clones that escaped shRNA-mediated Ezh2 knockdown did either not contain vector sequences or expressed vector sequences at very low levels (Supplementary Fig. S4A, B). Additionally, all iPS cell clones expressed Ezh2 mRNA at levels, which were similar to those in 4F iPS cells without shEzh2 or with shLuc control, or in ES cells. There was no shRNAmediated knockdown of Ezh2 in all shRNA iPS cell clones analyzed, whereas we observed efficient shRNA-mediated knockdown of Ezh2 in MEF control (Supplementary Fig. $\mathrm{S} 4 \mathrm{C})$. Thus, there are apparently two reasons for escape of
Ezh2 knockdown: (i) the lack of successful infection with shEzh2 vector and (ii) low and most probably insufficient expression of shEzh2 vector. Taken together, knockdown of Ezh2 severely impaired iPS cell generation, which shows that Ezh2 is required for efficient iPS cell generation.

We then proceeded to investigate whether Ezh2 also impacts on iPS cell self-renewal. shEzh2-1 and shEzh2-2 caused effective knockdown of Ezh2 in iPS cells and ES cells, and a reduction in $\mathrm{H} 3 \mathrm{~K} 27 \mathrm{me} 3$ and an increase of the PRC2 target gene Gata4 (Supplementary Fig. S5A, B). Yet knockdown of Ezh2 did not affect (i) the characteristic colony morphology of iPS cells and ES cells, (ii) expression of the pluripotency markers Oct4 and SSEA1, and (iii) proliferation of iPS cells and ES cells (Supplementary Fig. S5B-D, and data not shown). Thus, Ezh2 is dispensable for ES cell and iPS cell self-renewal in culture.

\section{Ezh2 acts in iPS cell generation through repressing the Ink4a/Arf locus}

Ezh2 represses the Ink4a/Arf locus, which encodes the cell cycle kinase (CDK) inhibitors p16Ink4a and p19Arf [37]. In addition, Ezh2 overexpression allows MEF to bypass senescence by repressing the Ink4a/Arf locus (Supplementary Fig. S6A-C) $[35,37]$. During iPS cell generation, the Ink4a/Arf locus is epigenetically reprogrammed toward a bivalent silent configuration with H3K27me3 and H3K4me3 marks, which leads to Ink4a/Arf repression [30].

Ezh2 knockdown increases Ink4a expression in ES cells and iPS cells and during iPS cell generation (Supplementary Fig. S7A, B). Therefore, we investigated whether Ink4a/Arf knockdown can rescue the impaired iPS cell generation by Ezh2 knockdown and performed simultaneous Ezh2 and Ink4a/Arf knockdown experiments. MEF were cotransfected with reprogramming factors, OSKM and Ezh2 shRNA, and with or without shRNA targeting Ink4a/Arf. Efficiency of reprogramming was measured at day 10 by AP staining
A
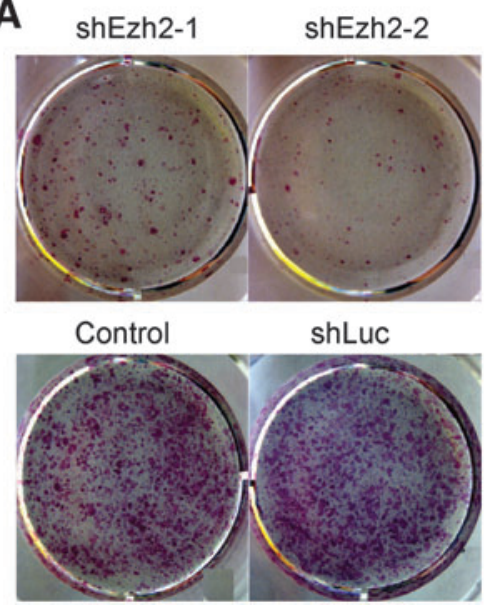
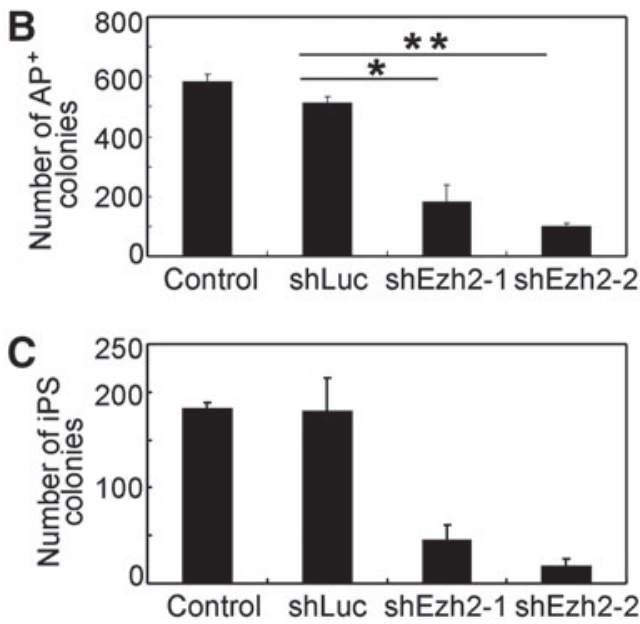

FIG. 4. Knockdown of Ezh2 expression impairs iPS cell generation. (A) Alkaline phosphatase (AP) staining during iPS cell generation (day 10). MEF were transduced with the four reprogramming factors OSKM together with Ezh2-1 or Ezh2-2 shRNA (shEzh2-1 or shEzh2-2, respectively), or Luc shRNA control (shLuc), or were left untreated (Control). Cells were maintained in the ES cell medium. $\mathrm{AP}^{+}$colonies are shown in red. One representative of three independent experiments is shown. (B) Quantification of $\mathrm{AP}^{+}$colonies (day 10). The number of $\mathrm{AP}^{+}$colonies in (A) was determined and is shown as mean value of three independent experiments $(* P<0.05$; $* * P<0.01)$. (C) Numbers of iPS cell colonies were scored by morphology at day 20 after infection. Results of three independent experiments are shown. 

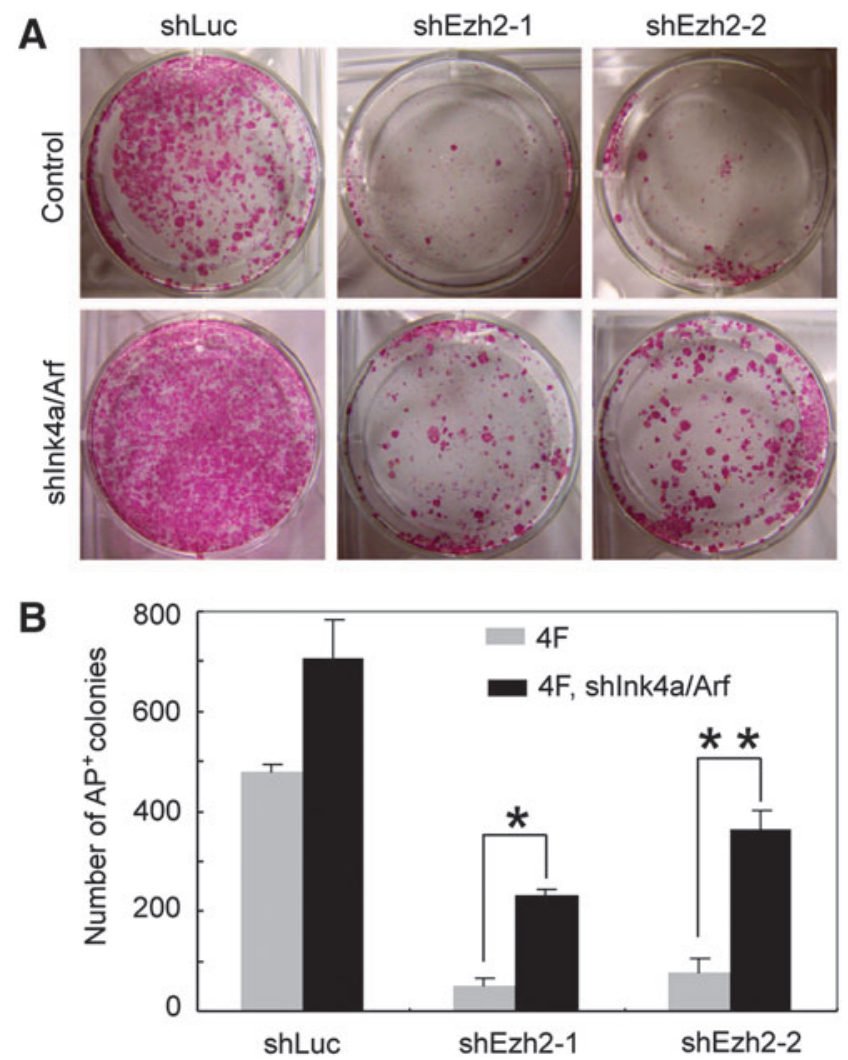

FIG. 5. Ezh2 impacts on iPS cell generation in part through repressing Ink4a/Arf. (A) shRNA knockdown of Ink4a/Arf (shInk4a/Arf) increases the frequencies of $\mathrm{AP}^{+}$ colonies during iPS cell generation. MEF were transduced with the OSKM reprogramming factors and Ezh2 shRNA (shEzh2-1 and shEzh2-2) plus shRNA against Ink4a/Arf (shInk4a/Arf). Cells were maintained in the ES cell medium, and AP staining was performed at day 10. One representative of four independent experiments is shown. (B) Numbers of $\mathrm{AP}^{+}$colonies in (A) were calculated and are shown as means of three independent experiments $(* P<0.05$; $* * P<0.01)$. Efficient Ezh2 knockdown in individual double knockdown iPS cell clones was shown by qRT-PCR analysis (data not shown).

(Fig. 5A, B). In accord with published data [30], we observed a higher frequency of $\mathrm{AP}^{+}$colonies with OSKM and shInk4a/ Arf. As expected, Ezh2 knockdown increased Ink4a expression and impaired iPS cell generation (Supplementary Fig. S7B and Fig. 4A, B), and this was partially rescued by simultaneous knockdown of Ink4a/Arf expression (Fig. 5A, B).

\section{Discussion}

In this study, we examined the impact of the $\mathrm{PcG}$ protein Ezh2 on iPS cell generation. We found that Ezh2 is induced during four-factor mediated reprogramming of $\mathrm{MEF}$ and that iPS cells contain high levels of Ezh2 protein, which are similar to the levels in ES cells. Importantly, Ezh2 knockdown severely impaired iPS cell generation, and thus, Ezh2 upregulation is critical for efficient reprogramming. We also found that Ezh2 impacts on iPS cell generation at least in part through repression of the CDK inhibitor Ink4a/Arf, which represents a major roadblock for iPS cell generation.
Somatic cell reprogramming and induction of pluripotency involve extensive chromatin reorganisation $[15,16]$, and enforced expression of subunits of the chromatin remodeling BAF complex facilitates iPS cell generation [21]. Additionally, expression of Trithorax complex component WD repeat domain 5 (Wdr5), an effector of histone $\mathrm{H} 3$ lysine 4 (H3K4) methylation, is required for efficient iPS cell generation [38]. Furthermore, inhibition of the H3 lysine 79 (H3K79) methyltransferase DOT1L accelerates reprogramming and increases the frequency of iPS cells [22]. Thus, all these findings demonstrate the impact of epigenetic modifiers as cofactors of the OSKM reprogramming factors to repress somatic gene expression and to activate the pluripotency program $[15,16,39]$.

Previous work showed that Ezh2 is important for establishing ES cell lines from blastocysts [7]. Additionally, Ezh2 was required for efficient somatic cell reprogramming by cell fusion and nuclear transfer $[9,10]$. These findings are in line with the results reported here. Ezh2 is abundantly expressed in iPS cells, and Ezh2 knockdown severely impaired iPS cell generation. However, once pluripotency is established, Ezh2 knockdown leaves the pluripotent phenotype of iPS cells unaffected. Additionally, as expected, Ezh2 knockdown reduced H3K27 trimethylation. But some H3K27me3 remained, probably due to compensation by other methyltransferases, such as Ezh1. Functional redundancy between Ezh1 and Ezh2 was described before [8,40]. All this indicates that Ezh2 is critical for induction of pluripotency, but once pluripotency is established, Ezh2 is not required anymore. The Ink4a/Arf locus represents a major target of Ezh2 [37] and repression of Ink4a/Arf improves iPS cell generation, and low Ink4a/Arf expression represent a feature of iPS cells and ES cells [30,41]. Thus, it appears conceivable that in established iPS cells Ink4a/Arf is silenced by mechanisms other than involving Ezh2. Yet, proper differentiation of iPS cells requires Ezh2 [42].

In a recent paper, Fragola et al. [24] generated iPS cells from $\mathrm{MEF}$ with a conditional $E z h 2$ knockout allele for the deletion of the catalytic Ezh2 SET domain. A cellpermeable TAT-Cre recombinase was used to inactivate Ezh2. Ezh2-deficient iPS cells were isolated, which exhibited a global loss of H3K27me3, yet a typical iPS cell phenotype, including ES cell-like morphology, growth, and differentiation potential. These findings contrast previous reports on Ezh2-deficient blastocyts to produce no or very few ES cells and on Ezh2-deficient ES cells to fail in cell fusion-induced reprogramming of somatic cells toward pluripotency [7-9]. The results by Fragola et al. are also in contrast to the shRNA knockdown studies reported here on primary iPS cells and on a doxycylin-inducible secondary iPS cell system [17]. Additionally, it also contrasts findings by the same authors on shRNA-induced knockdown of the PRC2 component EED, which reduced iPS cell generation by over $80 \%$ [24]. Thus, the result on Ezh2-deficient iPS cells might be rather methodically, in that Ezh2 inactivation could have occurred after reprogramming. Such an interpretation would be in line with the results reported here on Ezh2 knockdown in established pluripotent cells, which leaves morphology, pluripotency, and self-renewal of ES cells and iPS cells unaffected.

PcG components show cell type-specific expression pattern [1-3] and, as shown here, Ezh2 is abundantly expressed in ES cells and iPS cells. Elevated levels of Ezh2 expression 
have also been reported in tumor cells [43,44]. Additionally, c-Myc, one of the iPS cell inducing factors, was recently shown to directly regulate the Ezh2 expression and to be required for maintaining high Ezh2 expression in ES cells [45]. So far, among the four reprogramming factors, only c-Myc can efficiently induce Ezh2 expression. Yet, as shown here, enforced Ezh2 expression enhanced reprogramming efficiency only to some extent and could not compensate for the function of c-Myc in iPS cell generation.

The PRC1 component Bmi1 is an essential regulator for adult stem cells rather than ES cells [46]. In our previous work, we showed that forced Bmil expression promotes hematopoietic cell development from ES cells [26]. We also found that Bmil can efficiently substitute for c-Myc in iPS cell generation (unpublished data). In line with this, a recent study demonstrated that forced Bmil expression caused transdifferentiation of mouse fibroblasts into NSC-like cells and enhanced reprogramming efficiency and iPS cell generation [47]. However, enhanced iPS cell generation by enforced Bmil expression might be due to additional functions of Bmil, besides of being a component of PRC1 [48]. All this indicates a differential impact of chromatin modifying enzymes, including Ezh2 and Bmi1, on iPS cell generation. In addition, the H3K39 demethylase Jhdmla/lb represent yet another chromatin modifier that impacts on iPS cell generation [49].

Ezh2 is known as a senescence-preventing gene [37]. MEF undergo senescence in vitro, concomitantly with Ezh2 downregulation, loss of $\mathrm{H} 3 \mathrm{~K} 27 \mathrm{me} 3$ of the Ink4a/Arf locus, Ink4a/Arf activation, and cell cycle arrest [37]. Here, we found that during iPS cell generation, Ezh2 expression is induced and iPS cells contain high levels of Ezh2 protein. Additionally, Ink4a/Arf, a major target of Ezh2 in adult cells, has been identified as a roadblock of iPS cell generation $[30,41]$. Thus, we propose that during reprogramming and induction of pluripotency, Ezh2 upregulation and thus H3K27me3 efficiently represses Ink4a/Arf, which is crucial for efficient iPS cell generation. This notion is further supported by our observation that Ezh2 knockdown increases Ink4a/Arf expression and impairs iPS cell generation. Notably, Ink4a/Arf knockdown at least partially resuscitates the impaired iPS cell generation by Ezh2 downregulation. Our results therefore suggest that a major activity of the Ezh2/ Ink4a pathway on iPS cell generation might be to counteract senescence and thereby to affect the number of cells that are susceptible to reprogramming. The findings reported here extend previous knowledge of epigenetic modifiers impacting on iPS cell generation and highlight the intrinsic connection of reprogramming factors, epigenetic modifiers, and cell cycle regulators during the reprogramming process.

There is a significant variability of individual iPS cell clones in their differentiation potential. This is related to the (i) target cells, (ii) reprogramming factors and vector systems used for reprogramming, and (iii) further so far largely unknown factors [50]. In ES cells, many genes are marked with $\mathrm{H} 3 \mathrm{~K} 27 \mathrm{me} 3$, which is critical for their differentiation potential [10]. In addition, ES cells with disrupted PcG protein expression and consequently altered H3K27me3 show impaired differentiation potential [5,51]. Similarly, a recent study showed that Ezh2-null iPS cells exhibit severe defects in differentiation [42]. Thus, Ezh2 level might represent an important marker for evaluating iPS cell quality.
In summary, the data presented here demonstrate that Ezh2 impacts on iPS cell generation and acts at least in part through repressing the Ink4a/Arf locus, a major roadblock for iPS cell generation. The study emphasizes the importance of chromatin remodeling in cellular reprogramming toward pluripotency. In addition, it adds to our understanding of the underlying molecular mechanisms ongoing during iPS cell induction.

\section{Acknowledgments}

We thank Drs. A. Iwama and M. Serrano for plasmids. We also thank Andrea Offergeld and Renate Sous for expert secretarial assistance. This work was supported in part by grants from the Interdisciplinary Center for Clinical Research IZKF Aachen within the Faculty of Medicine, RWTH Aachen University, and DFG SPP1356 to M.Z. X.W. is a recipient of a fellowship of China Scholarship Council (CSC).

\section{Author Disclosure Statement}

No competing financial interestes exist.

\section{References}

1. Margueron R and D Reinberg. (2011). The Polycomb complex PRC2 and its mark in life. Nature 469:343-349.

2. Sauvageau M and G Sauvageau. (2010). Polycomb group proteins: multi-faceted regulators of somatic stem cells and cancer. Cell Stem Cell 7:299-313.

3. Schuettengruber B, D Chourrout, M Vervoort, B Leblanc and G Cavalli. (2007). Genome regulation by polycomb and trithorax proteins. Cell 128:735-745.

4. Ringrose L and R Paro. (2004). Epigenetic regulation of cellular memory by the Polycomb and Trithorax group proteins. Annu Rev Genet 38:413-443.

5. Boyer LA, K Plath, J Zeitlinger, T Brambrink, LA Medeiros, TI Lee, SS Levine, M Wernig, A Tajonar, et al. (2006). Polycomb complexes repress developmental regulators in murine embryonic stem cells. Nature 441:349-353.

6. Lee TI, RG Jenner, LA Boyer, MG Guenther, SS Levine, RM Kumar, B Chevalier, SE Johnstone, MF Cole, et al. (2006). Control of developmental regulators by Polycomb in human embryonic stem cells. Cell 125:301-313.

7. O'Carroll D, S Erhardt, M Pagani, SC Barton, MA Surani and T Jenuwein. (2001). The polycomb-group gene Ezh2 is required for early mouse development. Mol Cell Biol 21:4330-4336.

8. Shen X, Y Liu, YJ Hsu, Y Fujiwara, J Kim, X Mao, GC Yuan and SH Orkin. (2008). EZH1 mediates methylation on histone H3 lysine 27 and complements EZH2 in maintaining stem cell identity and executing pluripotency. Mol Cell 32:491-502.

9. Pereira CF, FM Piccolo, T Tsubouchi, S Sauer, NK Ryan, L Bruno, D Landeira, J Santos, A Banito, et al. (2010). ESCs require PRC2 to direct the successful reprogramming of differentiated cells toward pluripotency. Cell Stem Cell 6:547-556.

10. Zhang M, F Wang, Z Kou, Y Zhang and S Gao. (2009). Defective chromatin structure in somatic cell cloned mouse embryos. J Biol Chem 284:24981-24987.

11. Takahashi K and S Yamanaka. (2006). Induction of pluripotent stem cells from mouse embryonic and adult fibroblast cultures by defined factors. Cell 126:663-676. 
12. Grskovic M, A Javaherian, B Strulovici and GQ Daley. (2011). Induced pluripotent stem cells_opportunities for disease modelling and drug discovery. Nat Rev Drug Discov 10:915-929.

13. Stadtfeld M and K Hochedlinger. (2010). Induced pluripotency: history, mechanisms, and applications. Genes Dev 24:2239-2263.

14. Hanna JH, K Saha and R Jaenisch. (2010). Pluripotency and cellular reprogramming: facts, hypotheses, unresolved issues. Cell 143:508-525.

15. Plath K and WE Lowry. (2011). Progress in understanding reprogramming to the induced pluripotent state. Nat Rev Genet 12:253-265.

16. Gaspar-Maia A, A Alajem, E Meshorer and M RamalhoSantos. (2011). Open chromatin in pluripotency and reprogramming. Nat Rev Mol Cell Biol 12:36-47.

17. Buganim Y, DA Faddah, AW Cheng, E Itskovich, S Markoulaki, K Ganz, SL Klemm, A van Oudenaarden and R Jaenisch. (2012). Single-cell expression analyses during cellular reprogramming reveal an early stochastic and a late hierarchic phase. Cell 150:1209-1222.

18. Polo JM, E Anderssen, RM Walsh, BA Schwarz, CM Nefzger, SM Lim, M Borkent, E Apostolou, S Alaei, et al. (2012). A molecular roadmap of reprogramming somatic cells into iPS cells. Cell 151:1617-1632.

19. Vierbuchen T and M Wernig. (2012). Molecular roadblocks for cellular reprogramming. Mol Cell 47:827-838.

20. Meissner A. (2010). Epigenetic modifications in pluripotent and differentiated cells. Nat Biotechnol 28:1079-1088.

21. Singhal N, J Graumann, G Wu, MJ Arauzo-Bravo, DW Han, B Greber, L Gentile, M Mann and HR Schöler. (2010). Chromatin-remodeling components of the BAF complex facilitate reprogramming. Cell 141:943-955.

22. Onder TT, N Kara, A Cherry, AU Sinha, N Zhu, KM Bernt, P Cahan, BO Marcarci, J Unternaehrer, et al. (2012). Chromatin-modifying enzymes as modulators of reprogramming. Nature 483:598-602.

23. Hansson J, MR Rafiee, S Reiland, JM Polo, J Gehring, S Okawa, W Huber, K Hochedlinger and J Krijgsveld. (2012). Highly coordinated proteome dynamics during reprogramming of somatic cells to pluripotency. Cell Rep 2: 1579-1592.

24. Fragola G, PL Germain, P Laise, A Cuomo, A Blasimme, F Gross, E Signaroldi, G Bucci, C Sommer, et al. (2013). Cell reprogramming requires silencing of a core subset of polycomb targets. PLoS Genet 9:e1003292.

25. Do JT and HR Schöler. (2004). Nuclei of embryonic stem cells reprogram somatic cells. Stem Cells 22:941-949.

26. Ding $\mathrm{X}, \mathrm{Q}$ Lin, $\mathrm{R}$ Ensenat-Waser, $\mathrm{S}$ Rose-John and $\mathrm{M}$ Zenke. (2012). Polycomb group protein Bmil promotes hematopoietic cell development from embryonic stem cells. Stem Cells Dev 21:121-132.

27. Ruau D, R Ensenat-Waser, TC Dinger, DS Vallabhapurapu, A Rolletschek, C Hacker, T Hieronymus, AM Wobus, AM Müller and M Zenke. (2008). Pluripotency associated genes are reactivated by chromatin-modifying agents in neurosphere cells. Stem Cells 26:920-926.

28. Kim JB, H Zaehres, G Wu, L Gentile, K Ko, V Sebastiano, MJ Arauzo-Bravo, D Ruau, DW Han, M Zenke and HR Schöler. (2008). Pluripotent stem cells induced from adult neural stem cells by reprogramming with two factors. Nature 454:646-650.

29. Wang L, Q Jin, JE Lee, IH Su and K Ge. (2010). Histone H3K27 methyltransferase Ezh2 represses Wnt genes to facilitate adipogenesis. Proc Natl Acad Sci USA 107: 7317-7322.

30. Li H, M Collado, A Villasante, K Strati, S Ortega, M Canamero, MA Blasco and M Serrano. (2009). The Ink4/Arf locus is a barrier for iPS cell reprogramming. Nature 460:1136-1139.

31. Dickins RA, K McJunkin, E Hernando, PK Premsrirut, V Krizhanovsky, DJ Burgess, SY Kim, C Cordon-Cardo, L Zender, GJ Hannon and SW Lowe. (2007). Tissue-specific and reversible RNA interference in transgenic mice. Nat Genet 39:914-921.

32. Aoki R, T Chiba, S Miyagi, M Negishi, T Konuma, H Taniguchi, M Ogawa, O Yokosuka and A Iwama. (2010). The polycomb group gene product Ezh2 regulates proliferation and differentiation of murine hepatic stem/progenitor cells. J Hepatol 52:854-863.

33. Landazuri N, M Gupta and JM Le Doux. (2006). Rapid concentration and purification of retrovirus by flocculation with Polybrene. J Biotechnol 125:529-539.

34. Gamper I, KR Koh, D Ruau, K Ullrich, J Bartunkova, D Piroth, C Hacker, P Bartunek and M Zenke. (2009). GAR22: a novel target gene of thyroid hormone receptor causes growth inhibition in human erythroid cells. Exp Hematol 37:539-548.

35. Kamminga LM, LV Bystrykh, A de Boer, S Houwer, J Douma, E Weersing, B Dontje and G de Haan. (2006). The Polycomb group gene Ezh2 prevents hematopoietic stem cell exhaustion. Blood 107:2170-2179.

36. Koch CM, K Reck, K Shao, Q Lin, S Joussen, P Ziegler, G Walenda, W Drescher, B Opalka, et al. (2013). Pluripotent stem cells escape from senescence-associated DNA methylation changes. Genome Res 23:248-259.

37. Bracken AP, D Kleine-Kohlbrecher, N Dietrich, D Pasini, G Gargiulo, C Beekman, K Theilgaard-Monch, S Minucci, BT Porse, et al. (2007). The Polycomb group proteins bind throughout the INK4A-ARF locus and are disassociated in senescent cells. Genes Dev 21:525-530.

38. Ang YS, SY Tsai, DF Lee, J Monk, J Su, K Ratnakumar, J Ding, Y Ge, H Darr, et al. (2011). Wdr5 mediates selfrenewal and reprogramming via the embryonic stem cell core transcriptional network. Cell 145:183-197.

39. Liang G and Y Zhang. (2013). Embryonic stem cell and induced pluripotent stem cell: an epigenetic perspective. Cell Res 23:49-69.

40. Ezhkova E, WH Lien, N Stokes, HA Pasolli, JM Silva and E Fuchs. (2011). EZH1 and EZH2 cogovern histone H3K27 trimethylation and are essential for hair follicle homeostasis and wound repair. Genes Dev 25:485-498.

41. Utikal J, JM Polo, M Stadtfeld, N Maherali, W Kulalert, RM Walsh, A Khalil, JG Rheinwald and K Hochedlinger. (2009). Immortalization eliminates a roadblock during cellular reprogramming into iPS cells. Nature 460:1145-1148.

42. Villasante A, D Piazzolla, H Li, G Gomez-Lopez, M Djabali and M Serrano. (2011). Epigenetic regulation of Nanog expression by Ezh2 in pluripotent stem cells. Cell Cycle 10:1488-1498.

43. Shi B, J Liang, X Yang, Y Wang, Y Zhao, H Wu, L Sun, Y Zhang, Y Chen, et al. (2007). Integration of estrogen and Wnt signaling circuits by the polycomb group protein EZH2 in breast cancer cells. Mol Cell Biol 27:5105-5119.

44. Sander S, L Bullinger, K Klapproth, K Fiedler, HA Kestler, TF Barth, P Moller, S Stilgenbauer, JR Pollack and T Wirth. (2008). MYC stimulates EZH2 expression by repression of its negative regulator miR-26a. Blood 112:4202-4212. 
45. Neri F, A Zippo, A Krepelova, A Cherubini, M Rocchigiani and S Oliviero. (2012). Myc regulates the transcription of the PRC2 gene to control the expression of developmental genes in embryonic stem cells. Mol Cell Biol 32:840-851.

46. Valk-Lingbeek ME, SW Bruggeman and M van Lohuizen. (2004). Stem cells and cancer; the polycomb connection. Cell 118:409-418.

47. Moon JH, JS Heo, JS Kim, EK Jun, JH Lee, A Kim, J Kim, KY Whang, YK Kang, et al. (2011). Reprogramming fibroblasts into induced pluripotent stem cells with Bmi1. Cell Res 21:1305-1315.

48. Pietersen AM and M van Lohuizen. (2008). Stem cell regulation by polycomb repressors: postponing commitment. Curr Opin Cell Biol 20:201-207.

49. Wang T, K Chen, X Zeng, J Yang, Y Wu, X Shi, B Qin, L Zeng, MA Esteban, G Pan and D Pei. (2011). The histone demethylases Jhdm1a/1b enhance somatic cell reprogramming in a vitamin-C-dependent manner. Cell Stem Cell 9:575-587.

50. Lister R, M Pelizzola, YS Kida, RD Hawkins, JR Nery, G Hon, J Antosiewicz-Bourget, R O’Malley, R Castanon, et al. (2011). Hotspots of aberrant epigenomic reprogram- ming in human induced pluripotent stem cells. Nature 471:68-73.

51. Pasini D, AP Bracken, JB Hansen, M Capillo and K Helin. (2007). The polycomb group protein Suz12 is required for embryonic stem cell differentiation. Mol Cell Biol 27: 3769-3779.

Address correspondence to: Prof. Martin Zenke Department of Cell Biology Institute for Biomedical Engineering RWTH Aachen University Medical School Pauwelsstrasse 30 Aachen 52074 Germany

E-mail: martin.zenke@rwth-aachen.de

Received for publication June 13, 2013 Accepted after revision December 10, 2013 Prepublished on Liebert Instant Online December 10, 2013 